\title{
CAUSTIC INJURY IN ADULTS - A STUDY FOR 3 YEAR PERIOD
}

\author{
J. Radenkova-Saeva ${ }^{1}$, A. Loukova ${ }^{1}$ and Ch. Tsekov $^{2}$ \\ ${ }^{1}$ Toxicology Clinic, University Hospital "N. I. Pirogov", Sofia, Bulgaria \\ ${ }^{2}$ Division of Surgery, National Transport Hospital, Sofia, Bulgaria
}

\begin{abstract}
Summary. The aim of the study is to present the results of a 3-year clinicoepidemiological investigation of caustic injury in adults. The study includes 43 patients with acute corrosive ingestion, hospitalized in the Toxicology Clinic, University Hospital "N. I. Pirogov", Sofia, Bulgaria, for the period 01.01. 2010-31.12.2012. The methods used include: clinical observation and examination, clinical laboratory, imaging, and psychiatric methods and tests. 43 patients between the ages of 22 and 82 with acute corrosive ingestions have been observed. Eleven were male (25.6\%) and 32 female (74.4\%). All ingestions were intentional. Alkaline agents were used by all of the patients. The severity of poisonings varied from moderate to extremely severe. Different complications were seen in $82 \%$ of the cases - severe bleeding, perforation, fistula orland stricture formation. Two of the patients have undergone surgical intervention - coloesophagoplastic - and have recovered completely. The motivation in different age groups was also studied. Psychiatric comorbidity occurred in patients as depressive and schizoaffective disorder, as well as existential crises. Acute corrosive ingestions by alkaline agents cause severe pathology. The severity and complex character of the injuries require good coordination between different medical specialists.
\end{abstract}

Key words: alkaline agents, intentional self-poisoning, corrosive ingestion

\section{INTRODUCTION}

orrosives are materials that can attack and chemically destroy exposed body tissues. They begin to cause damage as soon as they touch the skin, eyes, respiratory tract, and digestive tract. Ingestion of corrosives can lead to extensive damage to the gastrointestinal tract and serious systemic features that may lead to death. Late complications may include stricture formation, 
gastric outlet obstruction and oesophageal carcinoma. Acids and alkalis are the two primary types of agents most often responsible for caustic exposures. Common acids include hydrochloric acid, sulfuric acid, nitric acid, chromic acid, acetic acid and hydrofluoric acid. Common bases are ammonium hydroxide, potassium hydroxide (caustic potash) and sodium hydroxide (caustic soda). Acid ingestion causes coagulation necrosis. In this process, hydrogen $(\mathrm{H}+)$ ions desiccate epithelial cells producing an eschar. This process leads to edema, erythema, mucosal sloughing, ulceration and necrosis of tissues. Alkali ingestion causes liquefaction necrosis. This process includes protein dissolution, collagen destruction, fat saponification, cell membrane emulsification, submucosal vascular thrombosis and cell death. Both acids and alkalis cause fibrosis and cicatrization (stricture formation). The physical characteristics of the corrosive substances (fluid or solid form, gel or granules) might influence on the localization of the post-corrosive injury [1, 2].

The aim of the study is to present the results of a 3-year clinico-epidemiological investigation of caustic injury in adults. The authors present the features and severity of acute intentional self-poisoning by alkaline corrosive agents in the Toxicology Clinic, Emergency University Hospital "N. I. Pirogov", Sofia, Bulgaria. Attention is paid to the motivation of patients for carrying out the suicide attempt by means of corrosives agents.

\section{MATERIAL AND METHODS}

The study includes 43 patients with acute corrosive ingestion, hospitalized in the Toxicology Clinic for the period 01.01. 2010-31.12.2012. The patients were followed-up with regard to general condition, local damage, psychiatric state and complications. The methods used include: clinical observation and examination, clinical laboratory, imaging and psychiatric methods and tests. The authors discussed the clinical presentation, treatment and management of complications.

\section{RESULTS}

43 patients between the ages of 22 and 82 with acute corrosive ingestions have been observed. Eleven were male (25.6\%) and 32 female (74.4\%). All ingestions were intentional. Alkaline agents were used by all of the patients. Clinical presentation of corrosive injuries in the upper gastrointestinal tract depends on the physical state, type and quantity of the corrosive substance. After caustic ingestion all our patients complain on painful and burning mouth and throat, retrosternal chest and stomach pains, nausea, vomiting, often with bloody content. The symptoms develop immediately after caustic ingestion, or few hours after ingestion and they last days and weeks. We observed also hypersalivation, difficulty in swallowing with edema, ulceration or whitish plaques in the oral cavity, palatal mucosa and pharynx. 
The golden standard for determination of the grade and extent of the lesion is esophagogastroduodenoscopy, performed in the first 12-24 hours following corrosive ingestion. In all patients was carried out esophagogastroduodenoscopy. The severity of poisonings varied from moderate to extremely severe (II-III degree on early endoscopic examination). Treatment includes parenteral hydration and nutrition, $\mathrm{H} 2$-receptor antagonists or proton pump inhibitors, steroids, antibiotics, analgesics, spasmolytics, sedatives. Different complications were seen in $82 \%$ of the cases of our study - severe bleeding, perforation, fistula or/and stricture formation. Two of the patients have undergone surgical intervention - coloesophagoplastic - and have recovered completely (Fig. 1, 2, 3, 4, 5, 6).
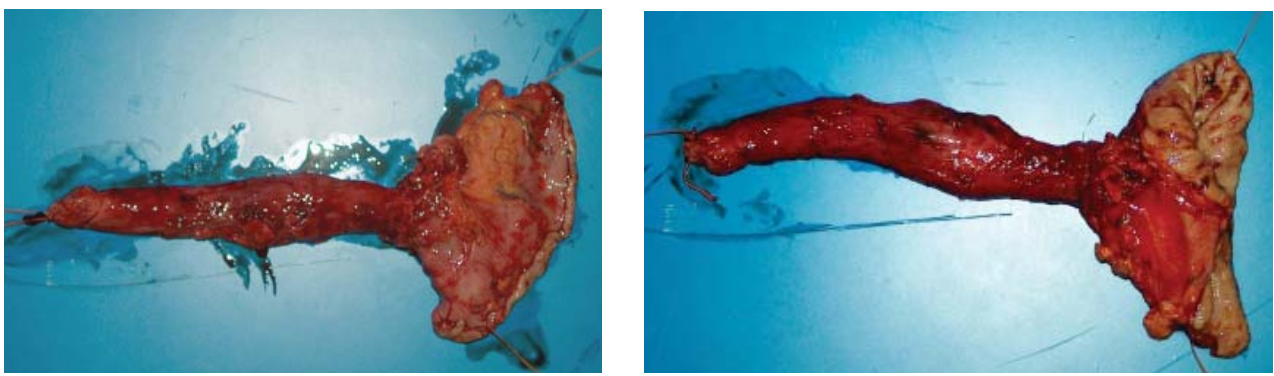

Fig. 1, 2. Extirpated oesophagus and stomach after postcorrosive damages
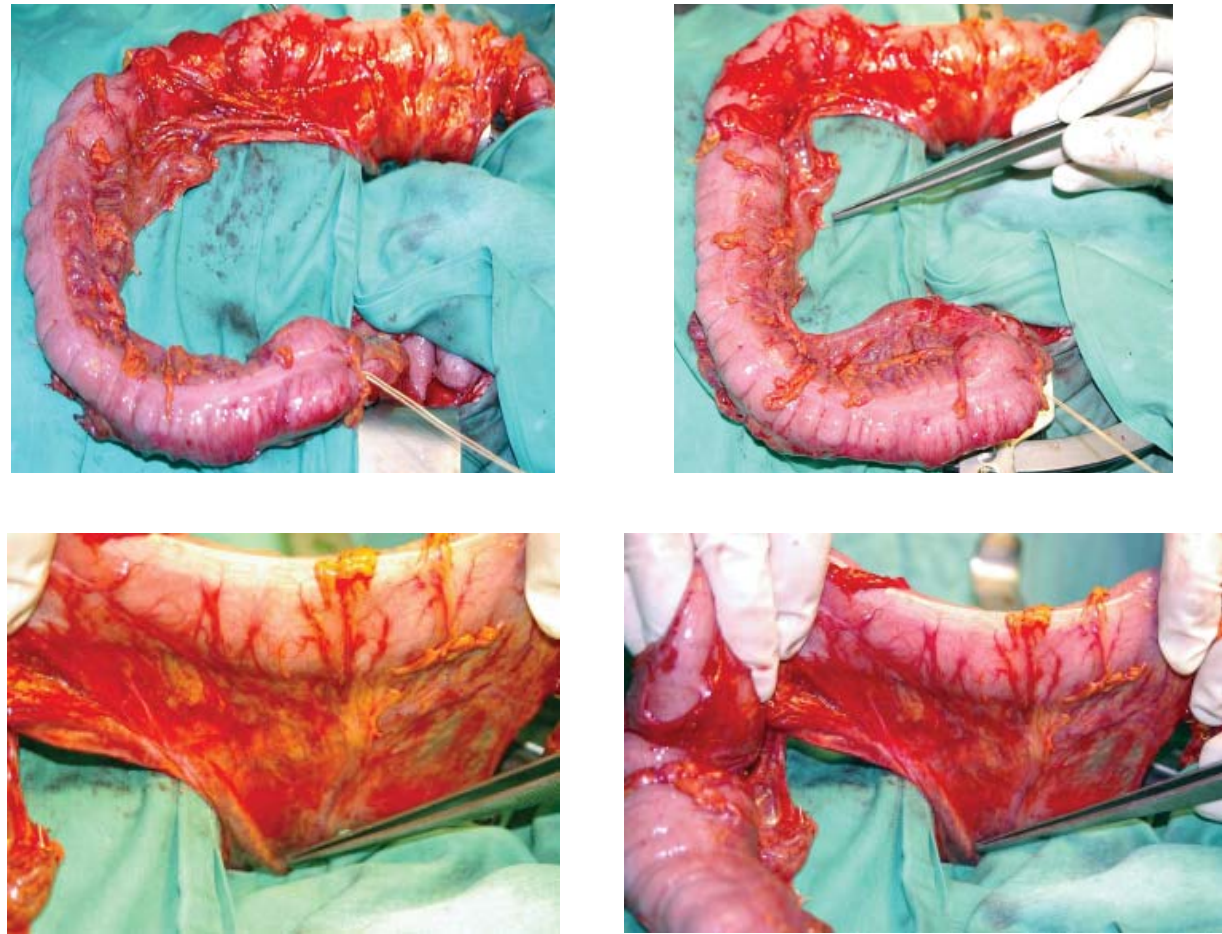

Fig. 3, 4, 5, 6. Different consecutive steps of oesophageal replacement with colon interposition 
Through an earnest psychiatric history, an exact mental status, allowing to establish the depth of mental disorder and implementation of various psychological tests as Questionnaire for assessment of suicidal risk; The Hamilton Rating Scale for Depression; The Mini Mental State Examination (MMSE), we observe significant correlation between severity of intoxication and patient's motivation. Psychiatric comorbidity occurred in patients as depressive and schizoaffective disorder, as well as existential crises. The analysis of the suicidal ingestions showed: women more often commit suicide by means of corrosives ingestion; the preferred corrosive substance is sodium hydroxide; the most frequent reasons for suicidal corrosive ingestions are existential crisis and depressive episode, followed by the major depressive episode; the patients with existential crisis are at the age 22-60; in the elder patients the leading reason for corrosive self-poisonings is depression. In this period of life the personality is forced to adapt to various changes different from their lifestyle (e.g. compulsory passiveness, loss of social status, financial instability and uncertainty, decrease social contacts, loss of friends and relatives, etc.). Thus new models of behavior emerge, enabling disadaptation and leading to suicide attempts.

\section{DISCUSSION}

Corrosive ingestions are serious medical problem in Bulgaria. The results from our study confirm the data, known from the literature and from the practice of the other countries. Ingestion of corrosive substance in a solid or gel form causes injuries at the level of the oropharynx and proximal segment of the esophagus, while corrosive liquid substances cause injuries on the middle and distal segments of the esophagus and stomach [1, 2].

Corrosive substance ingestion in the acute phase may result in injuries of the larynx and may cause laryngospasm associated with dyspnea, tachypnea, dysphonia and aphonia. Hypersalivation, difficulty in swallowing with edema, ulceration or whitish plaques in the oral cavity, palatal mucosa and pharynx are common phenomena. Aspiration of the corrosive substance may cause endotracheal or bronchial necrosis with mediastinitis, often leading to fatal outcome [3, 4].

Endoscopy has been called 'sine qua non' for evaluating patients with corrosive poisoning. Direct evaluation by endoscopy is useful in grading severity of tissue injury, planning for nutritional support and long-term management of strictures. Zargar's modified endoscopic classification of burns due to corrosive ingestion $[5,6]$.

They are graded as below:

Grade Description

- 0 - Normal mucosa

- 1 - Erythema/Hyperemia

- $2 a$ - Superficial ulcer/erosion/friability/hemorrhage/exudates

- $2 \mathrm{~b}$ - Findings in $2 \mathrm{a}+$ deep discrete/circumferential ulcers 
- $3 a$ - Scattered necrosis (black/grey discoloration)

- $3 b$ - Extensive/circumferential necrosis of mucosa

Upper gastrointestinal (GI) endoscopy should be done once the patient is hemodynamically stable and there are no signs of perforation. Urgent surgery is required in the event of perforation. Patients with Grade 2 and 3 injuries require intensive care [7].

Treatment of corrosive poisoning includes parenteral hydration and nutrition, $\mathrm{H} 2$ receptor antagonists or proton pump inhibitors. Any attempt at gastric emptying or dilution of compound is contraindicated in corrosive poisoning. Emetics should not be given as they increase the risk of mucosal injury and subsequent perforation. Nasogastric tube should not be inserted since it may cause esophageal perforation and increase the risk of aspiration. Dilution and neutralization of corrosive by nasogastric tube lavage generates heat and increases the risk of aspiration. Both have no proven benefit and hence are contraindicated. Stricture formation is the most severe late post-corrosive complication. Stricture can be prevented or reduced by steroid use $[8,9,10,11]$.

Tissue destruction from caustic injury increases the risk of infection by enteric organisms. That is why some authors justify the administration of broad spectrum of antibiotics, most commonly of the penicillin group [14].

The complications are a major problem in acute corrosive poisoning. The complications are acute, late and remote. Acute complications are shock, due to hemorrhage, vomiting or third-space sequestration; or GI perforation (can cause esophageal leak/rupture and mediastinitis or gastric leak/bleed leading to peritonitis). Late complications are strictures or obstruction. Remote complications include carcinoma of esophagus. Patients who develop esophageal strictures after alkali consumption have high-risk (1,000 times more risk than the general population) for the development of squamous cell carcinoma of esophagus. The mean latency period is 40 years after ingestion and in $84 \%$ of the patients the malignancy is located in the area of the bifurcation of trachea $[3,4,13]$.

Management of complications includes laparotomy, dilatation therapy and surgery. Laparotomy is indicated in patients with: endoscopic or radiologic evidence of perforation; severe abdominal rigidity; persistent hypotension; respiratory distress; ascites or pleural effusion; $\mathrm{pH}<7.2$ on arterial blood gas (ABG) analysis. Laparotomy permits tissue visualization, resection and repair of perforation. Stricture formation begins weeks to months after injury and is the most important consequence of corrosive poisoning. Procedures used for prevention and treatment of strictures are dilatation therapy and surgery: dilatation therapy is done 3-6 weeks after injury, progressively larger bougies are passed over endoscopically placed guide wires for dilatation. But, the risk of perforation, aspiration and dysphagia is high. Esophageal strictures resistant to dilatation therapy may require surgery that includes resection of stricture surgically and esophageal bypass surgery [14, 15, 16, 17]. 


\section{CONCLUSION}

Acute corrosive ingestions by alkaline agents may cause severe to serious injuries of the upper gastrointestinal tract and the poisoning can even result in death. The severity and complex character of the injuries require good coordination between different specialists as toxicologists, oto-rhino-laryngologists, surgeons, psychiatrists and psychologists and a multidisciplinary approach to any patient with acute corrosive ingestion.

\section{REFERENCES}

1. Bremholm L, Winkel R, Born P, Suku ML. Acute esophageal necrosis, Ugeskr Laeger. 2009; 171: 3282-3283.

2. Kardon E. Caustic ingestion, Emergency Medicine Toxicology. [homepage on the Internet] [updated 2010 may].

3. Satar S, Topal M, Kozaci N, Ingestion of caustic substances by adults: American J Of Therap. 2004; 11: 258-261.

4. Arevalo-Silva C, Eliashar R, Wohlgelernter J, Elidan J, Gross M. Ingestion of caustic substances: a 15-year experience. Laryngoscope. 2006; 116: 1422-1426.

5. Hao-Tsai Cheng, Chi-Liang Cheng, Cheng-Hui Lin, Jui-Hsiang Tang et all. Caustic ingestion in adults: The role of endoscopic classification in predictingoutcome. BMC Gastroenterol. 2008; 8: 1-7.

6. Zargar SA, Kochhar R, Nagar B, et al. Ingestion of corrosive acid. Gastroenterology, 1989; 97: 702.

7. Lupa M., et al., Update on the Diagnosis and Treatment of Caustic Ingestion The Ochsner Journal, 9:54-59, 2009.

8. Abakumov MM, Pinchuk TP, Il'iashenko LG. Is antisecretory therapy of patients with chemical burn of the esophagus mandatory? Khirurgiia Mosk. 2007; 1: 20-24.

9. Munoz Botero NA, Perez Cano AM, Rodriguez Herrera R, Rojas Gomez MP, Soler Paez FA. Nutrition therapy for adult patients with caustic injuries to gastrointestinal tract. Nutr Hosp. 2010; 25: 231-237.

10. Fulton J., R. S. Hoffman, Steroids in second degree caustic burns of the esophagus: A systematic pooled analysis of fifty years of human data: 1956-2006. Second Degree Caustic Burns of the Esophagus, Clinical Toxicology (2007) 45, 402-408.

11. Peclova D. Navratil, Do corticosteroids prevent oesophageal stricture after corrosive ingestion, Toxicol Rev. 2005; 24: 125-129.

12. Munoz-Bonerand N, Gornet JM, Diagnostic and therapeutic menagement of digestive caustic burns. J Chirug (Paris). 2002; 139: 72-76.

13. Chibishev A. Post-corrosive late complications in esophagus and stomach - role of the esophageal rest. Med Arh. 2010; 64: 320-323.

14. Dzhafarov ChM, Dzhafarov ECh. Surgical treatment of cicatricial strictureof the esophagus and stomach after chemical burn Khirurgiia (Mosk). 2007; (1): 25-28.

15. Kochhar R, Kochhar S. Endoscopic balloon dilation for benign gastric outlet obstruction in adults. World J Gastrointest Endosc. 2010; 2: 29-35.

16. Korolev MP, Fedorov LE, Treatment of patients with combined burn stricture of the esophagus and stomach, Vestn Khir IM II Grek. 2005; 164: 70-72.

17. Mamede RC, De Mello Filho FV Treatment of caustic ingestion: an analysis of 239 cases. Dis Esophagus. 2002; 15: 210-213.

Corresponding author:

Assoc. Prof. Julia Radenkova-Saeva, MD, PhD

Clinic of Toxicology, UMHATEM „N. I. Pirogov”

21 Totleben Blvd.

$\mathrm{Bg}-1606$ Sofia

e-mail: jrsaeva2@yahoo.co.uk 